\title{
Seasonal variation of microbial activity in soil and forest floor under three different fir plantations
}

\author{
Serdar Akburak \\ Istanbul University-Cerrahpasa, Faculty of Forest, Soil Science and Ecology Department, Sartyer, Istanbul \\ Corresponding author: sakburak@istanbul.edu.tr
}

\begin{abstract}
Microbial activity is one of the important processes for biochemical cycles in soil and forest floor of ecosystems. Because, some of the carbon dioxide and nutrients needed by plants are released during the microbial activity. In this study, the relationships between environmental factors (moisture, temperature, $\mathrm{pH}$, electric conductivity, $\mathrm{C}$, $\mathrm{N}, \mathrm{Na}, \mathrm{Ca}, \mathrm{Mg}, \mathrm{K}, \mathrm{P})$ and seasonal variations of microbial respiration, microbial biomass-C and metabolic quotient $(\mathrm{qCO} 2)$ in the forest floor and soil $(0-5 \mathrm{~cm})$ under three adjacent fir plantation plots (Abies nordmanniana ssp. bornmuelleriana Mattf. (Ab), Abies cilicica Carr. (Ac) and Abies nordmanniana ssp. nordmanniana Mattf (An)) are investigated in Atatürk Arboretum, located in Istanbul-Turkey. A bimonthly sampling (from May-2012 to March-2013) was carried out by collecting 54 samples for each soil and forest floor samples within each species. According to the results, soil microbial respiration (SMR) has a significantly lower value in $A_{b}$ plot. Although SMR and soil microbial biomass-C (SMBC) were correlated with moisture and temperature in $\mathrm{A}_{n}$ plot, they were correlated with nutrients in the other plots. In general, an increase in soil respiration rates was observed in autumn and early spring. Forest floor microbial respiration (FFMR), microbial biomass-C (FFMBC) and metabolic quotent $\left(\mathrm{qCO}_{2}\right)$ did not differ among the plots. The measured FFMR, FFMBC and $\mathrm{qCO}_{2}$ parameters were lower in autumn than spring. Forest floor microbial parameters were thought to be drived by the variation of nutrients quantities. As a result, the microbial processes in both soil and forest floor were changed with the effect of different factors, although there was no clear difference among the plots.
\end{abstract}

Keywords: Microbial respiration, Microbial biomass-C, $\mathrm{qCO}_{2}$, Abies

\section{Introduction}

Microorganism activities are one of the most important ecosystem components evaluated as an indicator of soil quality protection and sustainability (Kara and Bolat, 2008; Bolat et al., 2015; Oyedele et al., 2015). Microbial biomass and microbial respiration are considered as an index to evaluate soil microbial activity and health (Schoenholtz et al., 2000; Mariani et al., 2006).

Soil microbial biomass plays an essential role in soil fertility and nutrient retention in terrestrial ecosystems (Allen and Schlesinger, 2004) and serves as a resource of nutrients available for plant development. For instance, soil microbial biomass variations reflect immobilization levelsmineralization of nutrients (carbon and nitrogen) (Yang et al., 2010). Similarly, Oktay and Tecimen (2016) indicated that nitrogen mineralization in summer was close to zero and this fact could happen due to microbial immobilization. Microbial biomass is a viable component of soil organic matter, accounting for $1-5 \%$ of the total organic matter content (Jiang et al., 2009) and faster response to alterations in soil than soil organic matter (SOM) (Brookes et al., 2008; Araujo et al., 2010; Haripal and 
Sahoo, 2014). The chemical and physical properties of the soil can both directly and indirectly affect the distribution and structure of the decomposer community. Many studies have investigated the relationships among microbial community structure, activity and environmental factors such as moisture, temperature, $\mathrm{pH}$, nitrogen, carbon and nutrients (Na, Ca, P, Mg, S, etc.) (Diaz-Ravina et al., 1993; Priess and Fölster, 2001; Nsabimana et al., 2004; Pietri and Brookes, 2008; Pei et al., 2016; Soong et al., 2018; Wang et al., 2018). However, the precise and detailed mechanism of how the microbial community structure or activities respond to changes in environmental parameters is still uncertain (Singh, 2018).

Vegetation can directly influence the microbial activity and microbial biomass through the impact on microclimate and the amount and quality of the forest floor. Relatedly, Prescott and Grayston (2013) stated that vegetation characteristics affect the microbial activity and microbial community structure.

Due to the release of carbon dioxide and nutrients during the process (Glassman et al., 2018), litter decomposition is an important process for the nutrient cycle in soils of forest ecosystems. Litter decomposition rate is affected by abiotic (litter chemistry, nutrient presence, etc.) and biotic (soil microorganisms, fauna, etc.) factors (Wang et al., 2018). For example, low nutrient concentration in the forest floor can reduce microbial respiration and biomass (Priess and Fölster, 2001). In addition, Prescott and Grayston (2013) emphasized that examining the relationships between cations in the forest floor and the microbial community can be instructive in understanding the mechanisms underlying different nutrient cycles and microbial community structures under different tree species.

The Abies family (Pinaceae) is represented by two species and six subspecies in Turkey (Çakır, 2018). Abies nordmanniana ssp. bornmuelleriana Mattf., Abies nordmanniana ssp nordmanniana Mattf. and Abies cilicica Carr. are the most common abies species in Turkey. These species constitute to 670390 ha of entire forest area in Turkey (OGM, 2013) and are found in different regions such as Marmara, Mediterranean and Black Sea. In the sense of this study, it is crucial to state that these tree species were planted in Atatürk Arboretum in 1960. It is determined that the stands have the same site characteristics such as soil type, climate etc. (Çakır, 2018).

The aim of the study is to investigate the seasonal changes of microbial parameters (microbial respiration and microbial biomass carbon) in forest floor and soil under three fir species in the same site and their relationship with various environmental factors (moisture, temperature, $\mathrm{pH}$, electric conductivity, $\mathrm{C}, \mathrm{N}$, $\mathrm{Na}, \mathrm{Ca}, \mathrm{Mg}, \mathrm{K}, \mathrm{P})$.

\section{Material and Methods}

\section{Research Area}

The study area, Atatürk Arboretum, is located in Belgrad Forest in Istanbul, Turkey $\left(41^{\circ} 09^{\prime} 48^{\prime \prime}-\right.$ $41^{\circ} 10^{\prime} 55^{\prime \prime} \mathrm{N}$ and $28^{\circ} 57^{\prime} 27^{\prime \prime}-28^{\circ} 59^{\prime} 27^{\prime \prime} \mathrm{E}, 140 \mathrm{~m}$ asl.). According to the long-term data from the Bahçeköy Meteorology Station, the average annual temperature and precipitation is $13.0^{\circ} \mathrm{C}$ and 1121 $\mathrm{mm}$, respectively. Belgrad Forest has a maritime climate with moderate water deficiency in summers. The soil in the research area is Luvisol (WRB) and soil texture is loamy. The vegetative period lasts for 7.5 months (230 days) on average (Çakır, 2018).

\section{Field data}

Sampling was carried out in three adjacent fir plantation plots (Abies nordmanniana ssp. bornmuelleriana Mattf., Abies cilicica Carr. and Abies nordmanniana ssp nordmanniana Mattf). 
Sampling was performed at the central $40 \times 40 \mathrm{~m}$ plot within each plot to minimize the negative edge effects. The sample plots had homogeneity of abiotic environmental conditions (the aspect, slope, elevation and soil type). Sampling was carried out by collecting both 54 samples in soil and forest floor within each species ( 3 species $\times 3$ cores $\times 6$ dates $=54$ cores) on each sampling date (a bimonthly from May-2012 to March-2013). Soil samples were taken from the upper $5 \mathrm{~cm}$ of soil layer with steel soil cores with a $100 \mathrm{~cm}^{3}$ volume. The forest floor organic matter was collected from $0.25 \mathrm{~m}^{2}$ area. Three replicated samples (soil and forest floor) were taken systematically and composited on each plot.

\section{Chemical Measurements}

Soil samples were oven-dried at $105^{\circ} \mathrm{C}$ and entire roots and materials larger than $2 \mathrm{~mm}$ were removed by use of $2 \mathrm{~mm}$ sieve. Soil acidity (pH) and electrical conductivity (EC) were measured from 1:2.5 and 1:5 ratios of soil to deionized water soil slurry, respectively. Exchangeable cations ( $\mathrm{K}, \mathrm{Ca}, \mathrm{Mg}, \mathrm{Na}$ ) in $10 \mathrm{~g}$ soil samples were extracted with ammonium acetate solution $(1 \mathrm{~N}, \mathrm{pH}=7)$. Exchangeable $\mathrm{P}$ in 2 g samples were extracted with sodium bicarbonate solution $(0.5 \mathrm{M}, \mathrm{pH}=8.5)$. The concentrations of the individual cations were determined by ICP/OES (Perkin Elmer Optima DV7000) (Akburak et al., 2018).

The dry mass of forest floor samples was determined at $65{ }^{\circ} \mathrm{C}$ for $48 \mathrm{~h}$. Samples were digested with concentrated $\mathrm{HNO}_{3}\left(\%\right.$ 65) and $\mathrm{H}_{2} \mathrm{O}_{2}(\%$ 37) in a microwave oven (Berghoff Speedwave). The total concentration of elements $(\mathrm{P}, \mathrm{K}, \mathrm{Ca}, \mathrm{Mg}, \mathrm{Na}$ ) were measured by ICP/OES (Perkin Elmer Optima DV7000). The carbon (C) and nitrogen $(\mathrm{N})$ contents of soil and forest floor were determined with dry combustion method, using a LECO Truspec CN-2000 analyzer (Çakır and Akburak, 2017).

\section{Microbial parameters measurement}

To measure microbial respiration (MR), soil samples ( $20 \mathrm{~g}$ oven-dry soil equivalent) and forest floor samples ( $5 \mathrm{~g}$ oven-dry mass equivalent) were incubated for 7 days at $25^{\circ} \mathrm{C}$ in $500 \mathrm{ml}$ vessels and $10 \mathrm{ml}$ of sodium hydroxide $(1 \mathrm{M})$ was placed in the media to absorb the respired $\mathrm{CO}_{2}-\mathrm{C}$. The released $\mathrm{CO}_{2}-\mathrm{C}$ was determined by adding $\mathrm{BaCl}_{2}$ and later titrating with $1 \mathrm{M}$ hydrochloric acid in the end of every 7 days (Alef and Nannipieri, 1995). Microbial biomass-C (MBC) was determined by the substrate-induced respiration (SIR) method. SIR was acquired by adding $60 \mathrm{mg}$ glucose to soil samples (20 g oven-dry soil equivalent). The released $\mathrm{CO}_{2}$ was trapped in $0.05 \mathrm{M}$ sodium hydroxide for 4 -h incubation at 25 ${ }^{\circ} \mathrm{C}$ and measured by titration (Alef and Nannipieri, 1995). The metabolic quotient, $\mathrm{qCO}_{2}$, was calculated by dividing microbial respiration with microbial biomass carbon $\left(\mathrm{qCO}_{2}=\mathrm{MR} / \mathrm{MBC}\right)$ (Anderson and Domsch, 1986).

\section{Statistical analysis}

All data were tested for normal distribution using the Kolmogorov-Smirnov test. The data that did not accord with normal distribution were transformed with Box-Cox transformation. The significance of differences among tree species was tested by one-way analysis of variance (ANOVA) followed by Duncan test $(\mathrm{p}<0.05)$. In addition, correlations between microbial parameters and environmental variables were determined using the simple Pearson correlation coefficient. The statistical analyzes were performed in SPSS 21.0 package program.

\section{Results}

Soil

Soil N, Mg and $\mathrm{K}$ ratio were significantly lower in $\mathrm{A}_{\mathrm{b}}$ plot compared to other fir plots. However, $\mathrm{P}$ was significantly higher in $\mathrm{A}_{b}$ plot. Soil $\mathrm{C}$ and $\mathrm{C} / \mathrm{N}$ ratios in $\mathrm{A}_{c}$ plot were significantly higher $(\mathrm{p}<0.05)$ than the other two plots. Soil $\mathrm{pH}$ was significantly higher $(\mathrm{p}<0.05)$ in $\mathrm{A}_{\mathrm{n}}$ plot. 
The respiration rate ranged from $0.18-1.04 \mu \mathrm{g} \mathrm{CO}_{2}^{-} \mathrm{C} \mathrm{g}^{-} \mathrm{h}^{-}$in the $\mathrm{A}_{\mathrm{b}}, 0.36-0.72 \mu \mathrm{g} \mathrm{CO}_{2}-\mathrm{C} \mathrm{g}^{-} \mathrm{h}^{-}$in $\mathrm{A}_{\mathrm{c}}$ plot, and $0.47-1.01 \mu \mathrm{g} \mathrm{CO}_{2}-\mathrm{C} \mathrm{g}^{-} \mathrm{h}^{-}$in $\mathrm{A}_{\mathrm{n}}$ plot (Figure 1). SMR in the $\mathrm{A}_{\mathrm{n}}$ plot was significantly and 1.46 times higher in the $\mathrm{A}_{\mathrm{b}}$ plot, 1.33 times higher in the $\mathrm{A}_{\mathrm{c}}$ plot $(\mathrm{p}<0.05)$. Although $\mathrm{SMBC}$ and $\mathrm{SqCO}_{2}$ were at the lowest level in the $A_{b}$ plot, there were not statistical differences among the plots (Table 1).

Table 1: Chemical, physical and microbial properties of soil in the study plot

\begin{tabular}{|c|c|c|c|}
\hline & A. bornmuelleriana & A. cilicica & A. nordmanniana \\
\hline & Mean \pm Std.Er. & Mean \pm Std. Er & Mean \pm Std. Er \\
\hline $\operatorname{SMR}\left(\mu \mathrm{g} \mathrm{CO}_{2}-\mathrm{C} \mathrm{g}^{-} \mathrm{h}^{-}\right)$ & $0.49 \pm 0.07 \mathrm{a}$ & $0.54 \pm 0.04 \mathrm{~b}$ & $0.72 \pm 0.06 \mathrm{~b}$ \\
\hline SMBC (mg C g $\left.{ }^{-} h^{-}\right)$ & $0.27 \pm 0.03 \mathrm{a}$ & $0.32 \pm 0.04 \mathrm{a}$ & $0.31 \pm 0.03 \mathrm{a}$ \\
\hline $\mathbf{S q C O}_{2}\left(\mu \mathrm{g} \mathrm{CO}_{2}-\mathrm{C} \mathrm{mg} \mathrm{Ch} \mathrm{h}^{-}\right)$ & $1.84 \pm 0.22 \mathrm{a}$ & $1.95 \pm 0.24 \mathrm{a}$ & $2.40 \pm 0.22 \mathrm{a}$ \\
\hline $\mathbf{S N}^{*}(\%)$ & $0.17 \pm 0.01 \mathrm{~b}$ & $0.19 \pm 0.01 \mathrm{a}$ & $0.18 \pm 0.01 \mathrm{ab}$ \\
\hline $\mathbf{S C}^{*}(\%)$ & $5.28 \pm 0.55 \mathrm{~b}$ & $6.91 \pm 0.44 \mathrm{a}$ & $4.65 \pm 0.38 \mathrm{~b}$ \\
\hline SCN & $29.57 \pm 1.55 b$ & $35.37 \pm 1.15 \mathrm{a}$ & $25.33 \pm 1.31 \mathrm{c}$ \\
\hline $\mathbf{S M}^{*}(\%)$ & $28.75 \pm 2.81 \mathrm{a}$ & $34.96 \pm 3.07 \mathrm{a}$ & $31.59 \pm 3.10 \mathrm{a}$ \\
\hline $\mathbf{S T}^{*}\left({ }^{0} \mathrm{C}\right)$ & $16.08 \pm 1.24 \mathrm{a}$ & $16.69 \pm 1.29 \mathrm{a}$ & $16.02 \pm 1.26 \mathrm{a}$ \\
\hline $\mathbf{p H}^{*}$ & $5.15 \pm 0.08 \mathrm{a}$ & $5.08 \pm 0.08 \mathrm{a}$ & $5.58 \pm 0.03 \mathrm{~b}$ \\
\hline $\mathbf{E} \mathbf{C}^{*}(\mu \mathrm{S} \mathrm{cm})$ & $178.73 \pm 19.58 \mathrm{a}$ & $185.52 \pm 13.90 \mathrm{a}$ & $198.41 \pm 11.58 \mathrm{a}$ \\
\hline $\mathbf{C a}\left(\mathrm{mg} \mathrm{kg}^{-}\right)$ & $1177.21 \pm 66.45 \mathrm{a}$ & $1337.27 \pm 52.83 \mathrm{a}$ & $1357.92 \pm 70.06 \mathrm{a}$ \\
\hline $\mathbf{N a}\left(\mathrm{mg} \mathrm{kg}^{-}\right)$ & $17.40 \pm 1.50 \mathrm{a}$ & $24.41 \pm 2.10 \mathrm{~b}$ & $15.36 \pm 1.40 \mathrm{a}$ \\
\hline $\mathbf{K}\left(\mathrm{mg} \mathrm{kg}^{-}\right)$ & $88.93 \pm 7.90 \mathrm{~b}$ & $124.63 \pm 9.24 \mathrm{a}$ & $111.47 \pm 8.16 \mathrm{a}$ \\
\hline $\mathbf{M g}\left(\mathrm{mg} \mathrm{kg}^{-}\right)$ & $118.95 \pm 7.52 \mathrm{~b}$ & $237.32 \pm 30.07 \mathrm{a}$ & $198.13 \pm 22.61 \mathrm{a}$ \\
\hline $\mathbf{P}\left(\mathrm{mg} \mathrm{kg}^{-}\right)$ & $47.62 \pm 5.49 \mathrm{a}$ & $42.16 \pm 3.10 \mathrm{a}$ & $31.43 \pm 1.601 \mathrm{~b}$ \\
\hline
\end{tabular}

SMR : Soil microbial respiration, SMBC: Soil microbial biomass-Carbon, $\mathrm{SqCO}_{2}$ : Soil metabolic quotient, SN: Soil nitrogen, SC: Soil carbon, SCN: Carbon/nitrogen, SM: Soil moisture, ST: Soil temperature, pH: Soil acidity, EC: Soil electric conductivity, Ca: Calcium, Na: Sodium, K: Potassium, Mg: Magnesium, P: Phosphorous. Means with different letters in the same line are different $(\mathrm{P}<0.05)$.

“*” From Çakır (2018)

While SMR showed temporarily a parallel change among plots, an increase were observed for the respiration rates in autumn and early spring in all plots (Figure 1a). SMBC temporarily decreased from spring to autumn and reached the highest level in March in all plots (Figure 1b). $\mathrm{SqCO}_{2}$ showed a parallel change among all plots and reached its highest level in September (Figure 1c). Additionally, a high level of change for both SMR and $\mathrm{SqCO}_{2}$ was observed in $\mathrm{A}_{\mathrm{n}}$ plots (Figure 1a, c).

A positive relationship was found between $\mathrm{SMR}$ and $\mathrm{SMBC}, \mathrm{SqCO} 2, \mathrm{~N}, \mathrm{P}, \mathrm{EC}, \mathrm{SM}$ (soil moisture) in the $A_{b}$ plots, with $\mathrm{SqCO}_{2} \mathrm{ST}$ (soil temperature) in the $\mathrm{A}_{\mathrm{n}}$ plots. Whereas, there was only a significant positive relationship with $\mathrm{Na}$ and $\mathrm{K}$ in the $\mathrm{A}_{\mathrm{c}}$ plots $(\mathrm{p}<0.05)$. Additionally, a negative relationship was determined with SM in the $A_{n}$ plot and with $S C N$ in the $A_{c}$ plot (Table 2).

In terms of SMBC, there was a positive relationship with $\mathrm{EC}$ and $\mathrm{P}$ in $\mathrm{A}_{\mathrm{b}}$ plots, while the only significant one was between with $K$ in $A_{c}$ plots and with $S M$ in $A_{n}$ plots. However, while there was no negative relationship in $\mathrm{A}_{\mathrm{b}}$ plots, $\mathrm{SMBC}$ showed negative relation with $\mathrm{SqCO}_{2}$ and $\mathrm{C}$ in $\mathrm{A}_{\mathrm{c}}$ plot and with $\mathrm{SqCO} 2$, $\mathrm{SM}, \mathrm{EC}$ and $\mathrm{Ca}$ in $\mathrm{A}_{\mathrm{n}}$ plot (Table 2).

In terms of $\mathrm{SqCO}$, while there was no significant relationship in Ac plot, linear correlations were determined with N and SM (Ab plots) and ST and EC (An plot). In addition, An plot had a negative relationship with $\mathrm{SqCO} 2$ and $\mathrm{SM}$ (Table 2). 


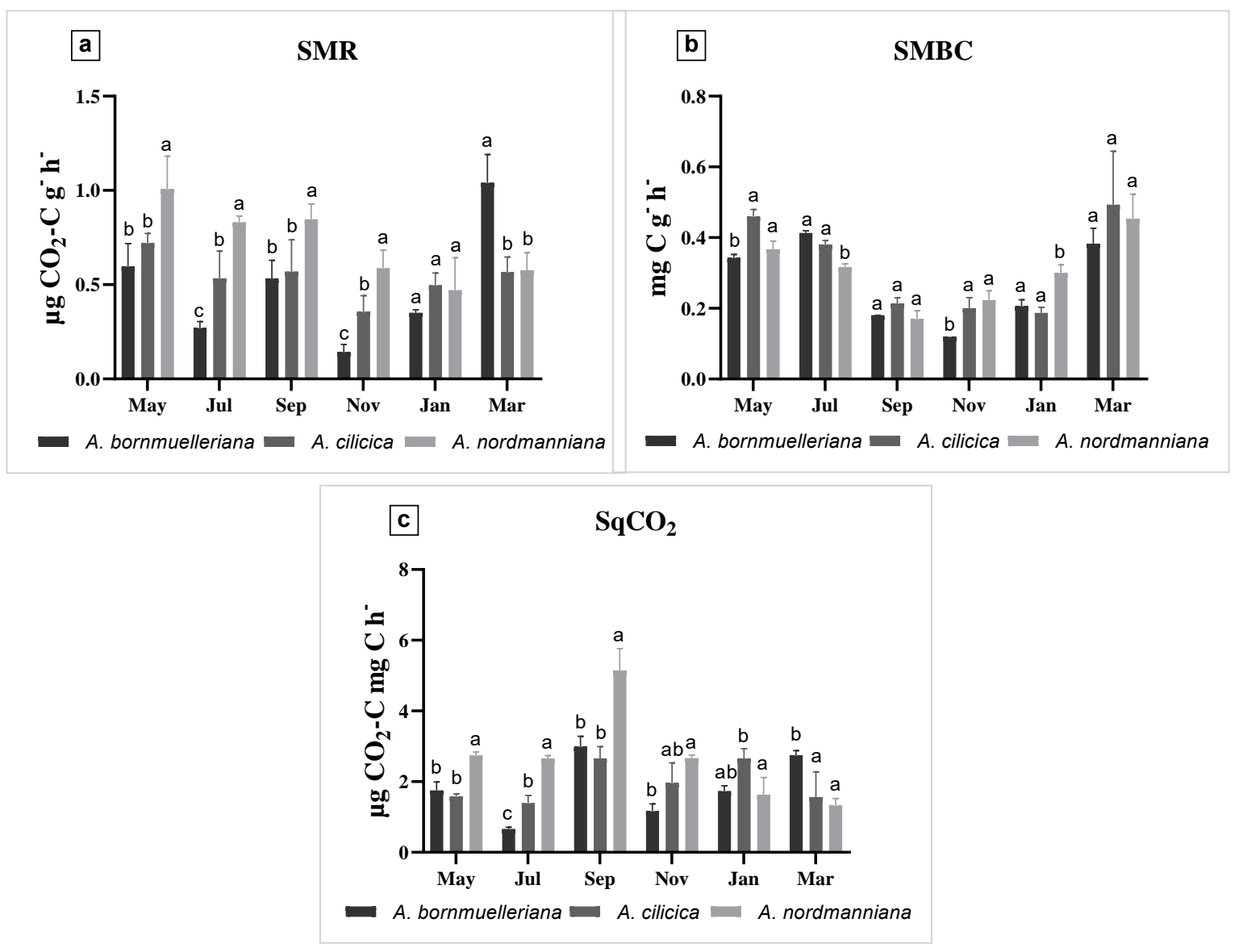

Figure 1: Seasonal variations of soil microbial parameter. (a: SMR; Soil microbial respiration, b: SMBC; Soil Microbial Biomass-C, c: qCO2; metabolic quotient).

\section{Forest floor}

In terms of FFMR, FFMBC, and $\mathrm{FFqCO}_{2}$, no significant differences were encountered among the plots. The mass of the forest floor (FF) in $A_{n}$ was significantly higher than the $A_{b}$ and $A_{c}(p<0.05)$ although in $A_{n}$, the FFC was significantly lower than the other plots (Table 3). K concentrations in $A_{b}$ plot were significantly different from $A_{c}$ and $A_{n}$ plots.

FFMR in all plots showed a decline in the autumn period (Figure 2a). FFMBC temporarily decreased from spring to autumn (Figure $2 \mathrm{~b}$ ). In addition, $\mathrm{FFqCO}_{2}$ showed a parallel change among all plots, reaching its highest level in November (Figure 2c).

According to relationships between the variables, the amount of FFMR in the $A_{b}$ plot showed a linear correlation with $\mathrm{FFMBC}, \mathrm{FFqCO}_{2}$ and $\mathrm{Ca}$, and a negative correlation with $\mathrm{Na}$. While there was a positive relationship with FFMBC and FM, a negative correlation was observed with $\mathrm{N}, \mathrm{C}, \mathrm{P}$ in the An plot. While it showed a positive relationship with FFMBC, FFM and a negative correlation with N, C, P in the $\mathrm{A}_{\mathrm{n}}$ plot was observed. In the $\mathrm{A}_{\mathrm{c}}$ plot, FFMR had a positive correlation with FFMBC and $\mathrm{FFqCO} 2$, and a negative relationship with $\mathrm{P}$ (Table 4).

In terms of FFMBC, there was no linear correlations in the $A_{b}$ and $A_{c}$ plots, while there was a linear correlation with $\mathrm{K}$ in the $\mathrm{A}_{\mathrm{n}}$. However, there were negative correlations with $\mathrm{Na}$ in $\mathrm{A}_{b}$ plot, with $\mathrm{P}$ in $\mathrm{A}_{c}$ plot and with $\mathrm{FFqCO}_{2}, \mathrm{Ca}$ in $\mathrm{A}_{\mathrm{n}}$ plots (Table 4). 
In respect of $\mathrm{FFqCO}_{2}$, there was a positive correlation with $\mathrm{Ca}$ and a negative correlation with $\mathrm{K}$ in the $\mathrm{A}_{b}$ plot. While it showed a negative correlation with FFN and $\mathrm{Mg}$ in the $\mathrm{A}_{\mathrm{c}}$ plot and $\mathrm{CN}, \mathrm{C}$ and $\mathrm{K}$ in the $\mathrm{A}_{\mathrm{n}}$ plot. Also, there was a linear correlation between $\mathrm{FFqCO}_{2}$ and $\mathrm{FF}$ in the $\mathrm{A}_{\mathrm{n}}$ plot (Table 4).

Table 2: Pearson correlation coefficients among the soil variables in the study

\begin{tabular}{|c|c|c|c|c|c|c|c|c|c|c|c|c|c|c|}
\hline A. bornmuelleriana & SMR & SMBC & $\mathrm{SqCO} 2$ & SN & $\mathrm{SC}$ & $\mathrm{SCN}$ & SM & ST & pH & EC & $\mathbf{C a}$ & $\mathrm{Na}$ & $\mathbf{K}$ & Mg \\
\hline SMBC & 0.48 & & & & & & & & & & & & & \\
\hline $\mathrm{SqCO2}$ & 0.73 & -0.22 & & & & & & & & & & & & \\
\hline SN & 0.59 & 0.29 & 0.48 & & & & & & & & & & & \\
\hline SC & 0.40 & 0.21 & 0.33 & 0.87 & & & & & & & & & & \\
\hline SCN & 0.24 & 0.15 & 0.17 & 0.68 & 0.94 & & & & & & & & & \\
\hline SM & 0.56 & -0.14 & 0.67 & 0.30 & 0.31 & 0.23 & & & & & & & & \\
\hline ST & -0.21 & 0.31 & -0.39 & 0.04 & 0.03 & 0.08 & -0.72 & & & & & & & \\
\hline pH & -0.06 & -0.13 & 0.05 & -0.11 & -0.08 & -0.02 & 0.12 & -0.18 & & & & & & \\
\hline EC & 0.49 & 0.47 & 0.24 & 0.68 & 0.71 & 0.67 & 0.17 & 0.16 & 0.28 & & & & & \\
\hline $\mathbf{C a}$ & -0.12 & 0.07 & -0.15 & 0.15 & 0.45 & 0.62 & -0.04 & 0.20 & 0.07 & 0.47 & & & & \\
\hline $\mathrm{Na}$ & 0.24 & 0.44 & -0.02 & 0.50 & 0.48 & 0.42 & -0.10 & 0.43 & -0.08 & 0.72 & 0.44 & & & \\
\hline $\mathbf{K}$ & 0.36 & 0.30 & 0.21 & 0.70 & 0.51 & 0.35 & -0.03 & 0.12 & -0.06 & 0.63 & 0.19 & 0.74 & & \\
\hline Mg & 0.18 & 0.27 & 0.07 & 0.47 & 0.57 & 0.63 & -0.14 & 0.33 & 0.01 & 0.72 & 0.79 & 0.65 & 0.60 & \\
\hline $\mathbf{P}$ & 0.58 & 0.49 & 0.24 & 0.63 & 0.67 & 0.61 & 0.40 & -0.07 & -0.33 & 0.52 & 0.29 & 0.42 & 0.30 & 0.36 \\
\hline A. cilicica & SMR & SMBC & $\mathrm{SqCO} 2$ & SN & SC & SCN & SM & ST & pH & EC & $\mathbf{C a}$ & $\mathrm{Na}$ & $\mathbf{K}$ & Mg \\
\hline SMBC & 0.39 & & & & & & & & & & & & & \\
\hline $\mathrm{SqCO2}$ & 0.22 & -0.81 & & & & & & & & & & & & \\
\hline SN & -0.15 & -0.37 & 0.33 & & & & & & & & & & & \\
\hline SC & -0.34 & -0.42 & 0.25 & 0.93 & & & & & & & & & & \\
\hline SCN & -0.46 & -0.40 & 0.14 & 0.77 & 0.95 & & & & & & & & & \\
\hline SM & 0.03 & -0.11 & 0.14 & -0.42 & -0.46 & -0.46 & & & & & & & & \\
\hline ST & 0.21 & 0.29 & -0.19 & 0.16 & 0.11 & 0.07 & -0.82 & & & & & & & \\
\hline pH & 0.12 & 0.40 & -0.32 & 0.34 & 0.26 & 0.18 & 0.03 & 0.04 & & & & & & \\
\hline EC & -0.22 & -0.29 & 0.19 & 0.72 & 0.65 & 0.51 & -0.30 & 0.16 & 0.31 & & & & & \\
\hline $\mathrm{Ca}$ & -0.18 & -0.15 & 0.08 & 0.27 & 0.30 & 0.30 & -0.03 & -0.10 & 0.10 & 0.21 & & & & \\
\hline $\mathrm{Na}$ & 0.47 & 0.26 & 0.01 & 0.25 & 0.16 & 0.12 & -0.46 & 0.49 & -0.01 & 0.15 & -0.05 & & & \\
\hline $\mathbf{K}$ & 0.53 & 0.44 & -0.11 & 0.24 & 0.06 & -0.04 & -0.19 & 0.26 & 0.35 & -0.05 & -0.27 & 0.67 & & \\
\hline Mg & 0.32 & 0.12 & 0.07 & 0.40 & 0.37 & 0.34 & -0.29 & 0.15 & 0.23 & -0.03 & -0.06 & 0.68 & 0.76 & \\
\hline $\mathbf{P}$ & -0.15 & -0.16 & 0.11 & 0.40 & 0.31 & 0.20 & -0.16 & -0.03 & -0.06 & 0.39 & 0.53 & -0.01 & -0.13 & -0.20 \\
\hline A. nordmanniana & SMR & SMBC & $\mathrm{SqCO2}$ & SN & SC & SCN & SM & ST & $\mathbf{p H}$ & EC & $\mathbf{C a}$ & $\mathbf{N a}$ & $\mathbf{K}$ & $\mathbf{M g}$ \\
\hline SMBC & -0.01 & & & & & & & & & & & & & \\
\hline $\mathrm{SqCO2}$ & 0.64 & -0.76 & & & & & & & & & & & & \\
\hline SN & 0.16 & 0.02 & 0.08 & & & & & & & & & & & \\
\hline SC & 0.22 & -0.32 & 0.34 & 0.66 & & & & & & & & & & \\
\hline SCN & 0.22 & -0.38 & 0.38 & 0.40 & 0.94 & & & & & & & & & \\
\hline SM & -0.50 & 0.44 & -0.65 & 0.00 & -0.38 & -0.50 & & & & & & & & \\
\hline ST & 0.72 & -0.29 & 0.69 & 0.03 & 0.36 & 0.45 & -0.90 & & & & & & & \\
\hline pH & -0.13 & 0.11 & -0.20 & 0.07 & 0.15 & 0.14 & 0.23 & -0.21 & & & & & & \\
\hline EC & 0.04 & -0.60 & 0.44 & 0.14 & 0.35 & 0.32 & -0.26 & 0.24 & 0.44 & & & & & \\
\hline $\mathbf{C a}$ & -0.27 & -0.57 & 0.22 & -0.02 & 0.36 & 0.44 & -0.15 & 0.04 & 0.08 & 0.51 & & & & \\
\hline $\mathrm{Na}$ & 0.24 & -0.10 & 0.21 & 0.14 & 0.34 & 0.33 & -0.07 & 0.24 & 0.48 & 0.59 & 0.23 & & & \\
\hline $\mathbf{K}$ & 0.27 & -0.30 & 0.37 & 0.26 & 0.47 & 0.41 & -0.24 & 0.40 & 0.40 & 0.73 & 0.26 & 0.73 & & \\
\hline Mg & 0.25 & -0.36 & 0.39 & 0.52 & 0.65 & 0.53 & -0.09 & 0.25 & 0.44 & 0.63 & 0.40 & 0.62 & 0.81 & \\
\hline $\mathbf{P}$ & 0.42 & 0.20 & 0.13 & 0.23 & 0.38 & 0.38 & -0.08 & 0.23 & 0.02 & -0.06 & -0.24 & 0.22 & 0.17 & 0.10 \\
\hline
\end{tabular}

SMR : Soil microbial respiration, SMBC: Soil microbial biomass-Carbon, $\mathrm{SqCO}_{2}$ : Soil metabolic quotient, SN: Soil nitrogen, SC: Soil carbon,

SCN: Carbon/nitrogen, SM: Soil moisture, ST: Soil temperature, pH: Soil acidity, EC: Soil electric conductivity, Ca: Calcium, Na: Sodium,

K: Potassium, Mg: Magnesium, P: Phosphorous. Significant differences $(P \leq 0.05)$ are marked with bold and italic 
Table 3: Chemical, physical and microbial properties of the forest floor in the study plot

\begin{tabular}{|c|c|c|c|}
\hline & A. bornmuelleriana & A. cilicica & A. nordmanniana \\
\hline & Mean \pm Std. Er & Mean \pm Std. Er & Mean \pm Std. Er \\
\hline FFMR $\left(\mu \mathrm{g} \mathrm{CO}{ }^{-}-\mathrm{C} \mathrm{g}^{-} \mathrm{h}^{-}\right)$ & $4.18 \pm 0.64 \mathrm{a}$ & $4.26 \pm 0.62 \mathrm{a}$ & $4.70 \pm 0.66 \mathrm{a}$ \\
\hline FFMBC $\left(\mathrm{mg} \mathrm{C} \mathrm{g}^{-} \mathrm{h}^{-}\right)$ & $2.40 \pm 0.28 \mathrm{a}$ & $1.96 \pm 0.24 \mathrm{a}$ & $2.37 \pm 0.31 \mathrm{a}$ \\
\hline $\mathbf{F F q C O}_{2}\left(\mu \mathrm{g} \mathrm{CO}{ }_{2}-\mathrm{C} \mathrm{mg} \mathrm{Ch} \mathrm{h}^{-}\right)$ & $1.99 \pm 0.36 \mathrm{a}$ & $2.22 \pm 0.27 \mathrm{a}$ & $2.39 \pm 0.36 \mathrm{a}$ \\
\hline $\mathbf{F F N * ( \% )}$ & $1.58 \pm 0.02 \mathrm{a}$ & $1.19 \pm 0.03 \mathrm{a}$ & $1.39 \pm 0.03 \mathrm{a}$ \\
\hline FFC* $(\%)$ & $42.96 \pm 0.46 \mathrm{a}$ & $41.92 \pm 0.88 \mathrm{a}$ & $37.09 \pm 1.19 \mathrm{~b}$ \\
\hline FFCN* & $27.32 \pm 0.43 \mathrm{ab}$ & $28.26 \pm 0.83 \mathrm{a}$ & $26.45 \pm 0.82 \mathrm{~b}$ \\
\hline $\mathbf{F F}^{*}\left(\mathrm{~g} \mathrm{~m}^{-2}\right)$ & $1027.02 \pm 49.97 \mathrm{a}$ & $1005.04 \pm 67.28 \mathrm{a}$ & $1452.86 \pm 84.61 \mathrm{~b}$ \\
\hline FFM $(\%)$ & $44.93 \pm 2.12 \mathrm{a}$ & $41.27 \pm 2.98 \mathrm{a}$ & $46.97 \pm 2.04 \mathrm{a}$ \\
\hline Ca $\left(\mathrm{mg} \mathrm{kg}{ }^{-}\right)$ & $14326.67 \pm 369.46 \mathrm{a}$ & $14650.83 \pm 567.98 \mathrm{a}$ & $14272.50 \pm 498.38 \mathrm{a}$ \\
\hline $\left.\mathbf{N a}(\mathrm{mg} \mathrm{kg})^{-}\right)$ & $54.08 \pm 5.26 \mathrm{a}$ & $68.23 \pm 8.30 \mathrm{a}$ & $52.57 \pm 4.46 \mathrm{a}$ \\
\hline $\mathbf{K}\left(\mathrm{mg} \mathrm{kg}^{-}\right)$ & $922.75 \pm 42.78 \mathrm{a}$ & $1081.77 \pm 34.37 \mathrm{~b}$ & $1072.517 \pm 50.21 \mathrm{~b}$ \\
\hline $\mathbf{M g}\left(\mathrm{mg} \mathrm{kg}^{-}\right)$ & $1193.82 \pm 46.69 \mathrm{a}$ & $1291.25 \pm 30.11 \mathrm{a}$ & $1339.583 \pm 42.89 \mathrm{a}$ \\
\hline $\mathbf{P}\left(\mathrm{mg} \mathrm{kg}^{-}\right)$ & $475.67 \pm 8.08 \mathrm{a}$ & $462.04 \pm 12.92 \mathrm{a}$ & $479.192 \pm 9.46 \mathrm{a}$ \\
\hline
\end{tabular}

FFMR : Forest floor microbial respiration, FFMBC: Forest floor microbial biomass-Carbon, $\mathrm{FFqCO}_{2}$ : Forest floor metabolic quotient, FFN: Forest floor nitrogen, FFC: Forest floor carbon, FFCN: Forest floor Carbon/nitrogen, FF: Forest floor amount, FFM: Forest floor moisture, Ca: Calcium, Na: Sodium, K: Potassium, Mg: Magnesium, P: Phosphorous. Means with different letters in the same line are different $(\mathrm{P}<0.05)$. “*” from Çakır (2018)

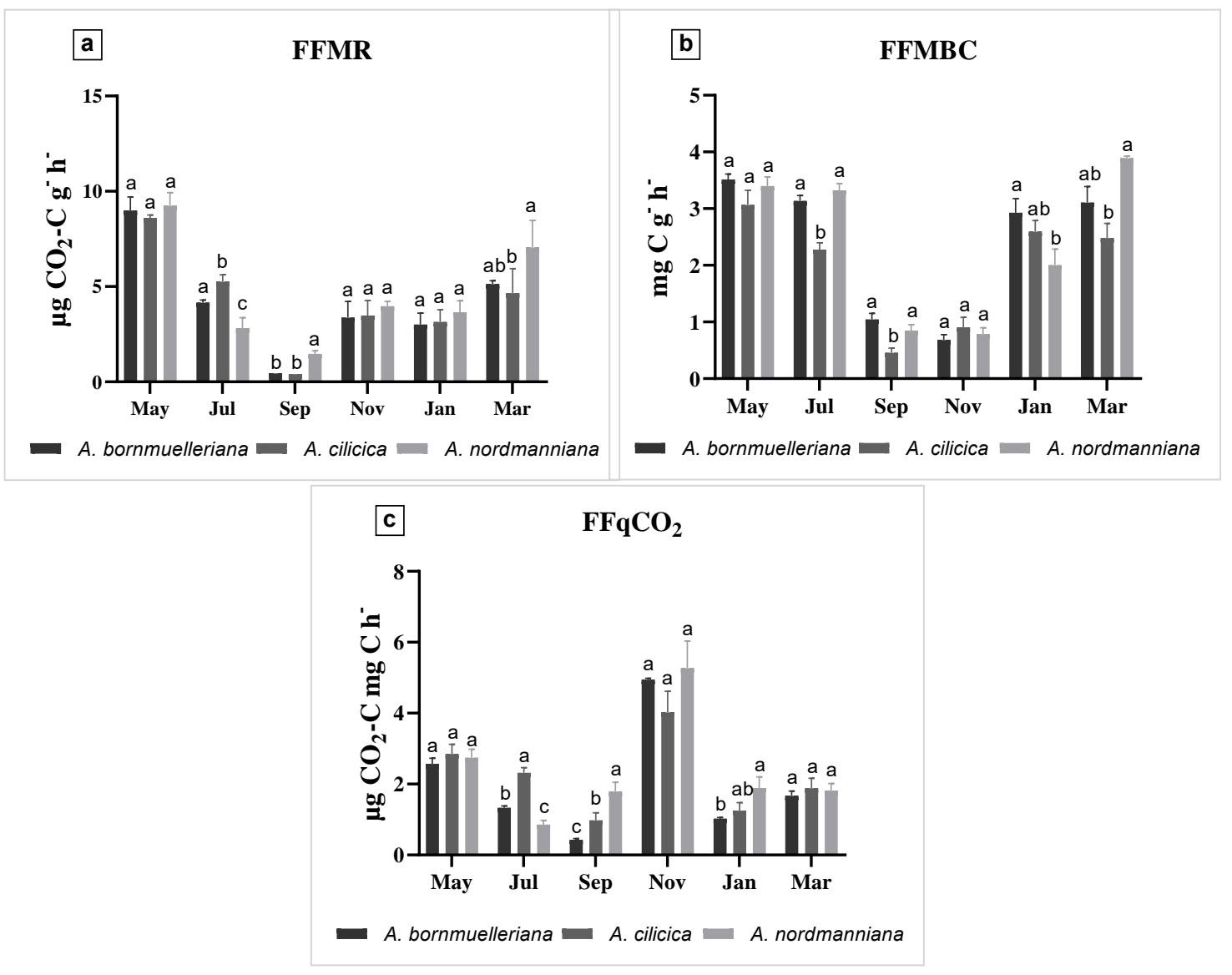

Figure 2: Seasonal variations of forest floor microbial parameters. (a: FFMR, Forest floor microbial respiration, b: FFMBC: Forest Floor Microbial Biomass-C, c: FFqCO2: Forest Floor metabolic quotient) 
Table 4: Pearson correlation coefficients among the forest floor variables in the study

\begin{tabular}{|c|c|c|c|c|c|c|c|c|c|c|c|c|}
\hline A. bornmuelleriana & FFMR & FFMBC & FFqCO2 & FFN & FFC & FFCN & $\mathbf{F F}$ & FFM & $\mathbf{C a}$ & $\mathrm{Na}$ & $\mathbf{K}$ & $\mathrm{Mg}$ \\
\hline FFMBC & 0.72 & & & & & & & & & & & \\
\hline $\mathrm{FFqCO2}$ & 0.63 & -0.02 & & & & & & & & & & \\
\hline FFN & 0.17 & 0.01 & 0.16 & & & & & & & & & \\
\hline FFC & 0.11 & 0.14 & 0.06 & 0.32 & & & & & & & & \\
\hline FFCN & -0.09 & 0.09 & -0.12 & -0.75 & 0.38 & & & & & & & \\
\hline FF & 0.24 & -0.08 & 0.26 & 0.34 & 0.03 & -0.34 & & & & & & \\
\hline FFM & 0.23 & 0.39 & -0.13 & -0.14 & -0.42 & -0.15 & 0.04 & & & & & \\
\hline $\mathrm{Ca}$ & 0.50 & 0.19 & 0.48 & -0.19 & 0.06 & 0.22 & 0.22 & -0.25 & & & & \\
\hline $\mathrm{Na}$ & -0.69 & -0.63 & -0.30 & -0.21 & -0.16 & 0.11 & -0.25 & -0.15 & -0.08 & & & \\
\hline $\mathbf{K}$ & -0.29 & 0.37 & -0.72 & -0.10 & -0.05 & 0.07 & -0.36 & 0.35 & -0.52 & 0.11 & & \\
\hline Mg & 0.04 & 0.15 & -0.18 & 0.31 & -0.28 & -0.49 & 0.11 & 0.32 & -0.22 & 0.02 & 0.49 & \\
\hline $\mathbf{P}$ & 0.38 & 0.15 & 0.19 & -0.21 & -0.05 & 0.20 & 0.07 & -0.17 & 0.63 & 0.14 & -0.35 & -0.03 \\
\hline A. cilicica & FFMR & FFMBC & FFqCO2 & FFN & FFC & FFCN & FF & FFM & $\mathbf{C a}$ & $\mathrm{Na}$ & $\mathbf{K}$ & Mg \\
\hline FFMBC & 0.76 & & & & & & & & & & & \\
\hline $\mathrm{FFqCO}$ & 0.46 & -0.08 & & & & & & & & & & \\
\hline FFN & -0.23 & 0.09 & -0.45 & & & & & & & & & \\
\hline FFC & 0.07 & 0.04 & -0.22 & -0.18 & & & & & & & & \\
\hline FFCN & 0.15 & -0.02 & 0.06 & -0.71 & 0.82 & & & & & & & \\
\hline FF & -0.23 & -0.31 & 0.29 & 0.04 & -0.72 & -0.57 & & & & & & \\
\hline FFM & 0.06 & 0.27 & -0.14 & 0.50 & -0.39 & -0.56 & 0.25 & & & & & \\
\hline $\mathrm{Ca}$ & 0.07 & -0.06 & -0.20 & 0.26 & 0.30 & 0.05 & -0.12 & -0.01 & & & & \\
\hline $\mathbf{N a}$ & -0.20 & -0.09 & -0.37 & 0.28 & 0.19 & -0.03 & 0.06 & -0.02 & 0.75 & & & \\
\hline $\mathbf{K}$ & -0.19 & -0.19 & -0.37 & 0.05 & 0.25 & 0.16 & -0.10 & -0.21 & 0.71 & 0.87 & & \\
\hline Mg & -0.22 & 0.07 & -0.46 & 0.66 & -0.03 & -0.36 & -0.26 & 0.34 & 0.13 & 0.09 & -0.03 & \\
\hline $\mathbf{P}$ & -0.50 & -0.50 & -0.38 & 0.48 & 0.09 & -0.19 & -0.10 & -0.03 & 0.70 & 0.54 & 0.55 & 0.48 \\
\hline A. nordmanniana & FFMR & FFMBC & FFqCO2 & FFN & FFC & FFCN & FF & FFM & $\mathrm{Ca}$ & $\mathrm{Na}$ & $\mathbf{K}$ & Mg \\
\hline FFMBC & 0.62 & & & & & & & & & & & \\
\hline $\mathrm{FFqCO}_{2}$ & 0.36 & -0.50 & & & & & & & & & & \\
\hline FFN & -0.47 & -0.27 & -0.24 & & & & & & & & & \\
\hline FFC & -0.50 & 0.06 & -0.60 & 0.45 & & & & & & & & \\
\hline FFCN & -0.26 & 0.26 & -0.53 & -0.14 & 0.81 & & & & & & & \\
\hline FF & 0.24 & -0.25 & 0.59 & -0.57 & -0.61 & -0.37 & & & & & & \\
\hline FFM & 0.46 & 0.11 & 0.35 & -0.27 & -0.43 & -0.24 & 0.02 & & & & & \\
\hline $\mathrm{Ca}$ & -0.36 & -0.48 & 0.26 & 0.00 & 0.18 & 0.25 & 0.29 & -0.18 & & & & \\
\hline $\mathbf{N a}$ & 0.02 & -0.15 & 0.15 & 0.23 & 0.12 & -0.03 & -0.18 & 0.52 & 0.02 & & & \\
\hline $\mathbf{K}$ & 0.11 & 0.43 & -0.46 & 0.14 & -0.02 & -0.17 & -0.27 & -0.31 & -0.81 & -0.41 & & \\
\hline Mg & 0.39 & 0.21 & 0.19 & -0.52 & -0.41 & -0.17 & 0.27 & -0.18 & -0.42 & -0.53 & 0.43 & \\
\hline $\mathbf{P}$ & -0.56 & -0.26 & -0.27 & 0.39 & 0.47 & 0.28 & -0.04 & -0.59 & 0.66 & -0.07 & -0.22 & -0.57 \\
\hline
\end{tabular}

FFMR : Forest floor microbial respiration, FFMBC: Forest floor microbial biomass-Carbon, $\mathrm{FFqCO}_{2}$ : Forest floor metabolic quotient, FFN: Forest floor nitrogen, FFC: Forest floor carbon, FFCN: Forest floor Carbon/nitrogen, FF: Forest floor amount, FFM: Forest floor moisture, Fe: Iron, Ca: Calcium, Na: Sodium, K: Potassium, Zn: Zinc, Cu: copper, Mg: Magnesium, Mn: Manganese, P: Phosphorous. Significant differences $(\mathrm{P} \leq 0.05)$ are marked with bold and italic

\section{Discussion}

\section{Soil}

Soil basal respiration provides an estimate of microbial activity (Bolat et al., 2015). The SMR rate obtained in the study sites ranged from 0.18 to $1.04 \mu \mathrm{g} \mathrm{CO} \mathrm{CO}^{-} \mathrm{C} \mathrm{g}^{-} \mathrm{h}^{-}$. These results are similar to those obtained with other studies (Hofman et al., 2004; Bolat, 2014; Bolat et al., 2015). SMR in Ab plot was significantly lower than other species. This may be due to the low amount of N, SM and SMBC in this plot compared to other fir species in the study site. Previous studies indicated that change of microbial respiration have relation with factors such as mineralized N, moisture and MBC (Priess and Fölster, 2001; Nsabimana et al., 2004).

Generally, a temporary increase in respiration rates was observed in fall and spring across all plots. As it was pointed out in Bolat et al. (2015), this increase might be a consequence of a promoting microbial 
activity which was triggered by higher soil temperature and moisture in these seasons. In addition, there were significant differences among the related species in July and November. Baldrian (2017) pointed out that seasonal variation is the most important driving force of microbial change with the soil temperature. When the interactions between SMR and variables are examined, it was seen that ST has a strong effect on SMR in the $A_{n}$ plot, while the presence of nutrients $\left(N, N a, K\right.$ and $P$ ) in the $A_{b}$ and $A_{c}$ plots were effective on SMR. As the results demonstrated in many studies, the microbial respiration is related to available nutrients (Cheng et al., 2013; Li et al., 2015; Spohn, 2015; Wang et al., 2018) and microbial biomass (Mariani et al., 2006; Bolat et al., 2015; Qu et al., 2018). Likewise, Spohn (2015) found that respiration have linear correlation with soil temperature. Wang et al. (2003) suggested that soil respiration under favorable temperature and moisture conditions was principally determined by substrate supply rather than by the pool size of MBC. As a result, it can be stated that different environmental factors have different effects on microbial process among plots.

The mean SMBC values were not showed significant differences among the plots. This may be due to the fact that soil properties (such as ST, SM and $\mathrm{pH}$ ) that affect SMBC do not differ between the plots. However, in terms of seasonal variations, SMBC decreased from spring to autumn and reached its highest level in all plots in March. Similar to the results of this study, Qu et al. (2018) stated that MBC was showed an increase early in the growing season (May) and then gradually decrease (July). This seasonal change may have two possible causes. The first one is the seasonal change in soil moisture and temperature. Second, the reduction in soil microbial biomass may be the result of mineralization of nutrients as stated by Jia et al. (2005). Soil microbial biomass is strongly affected by multiple factors such as local abiotic conditions and plant traits (Pei et al., 2016). Considering the relationship between SMBC and environmental variables, there is a positive relationship with $\mathrm{EC}$ and $\mathrm{P}$ in the $\mathrm{A}_{\mathrm{b}}$ plot, while there is a positive relationship only with $\mathrm{K}$ in the $\mathrm{A}_{c}$ plot, with $\mathrm{SM}$ and a negative relationship with EC and $\mathrm{Ca}$ in the $\mathrm{A}_{\mathrm{n}}$ plot. Previous studies showed that the microbial biomass is strongly dependent on soil properties such as exchangeable $\mathrm{Ca}$, and $\mathrm{pH}$ (Wolters and Joergensen, 1991; Agnelli et al., 2001). While the relationships between MBC and C, N were determined in the studies (Park et al., 2002; Mariani et al., 2006; Pei et al., 2016), no significant relationships were detected in presented study. Similar to current study results,Tan et al. (2008), Pei et al. (2016), Docherty et al. (2015) and Cheng et al. (2013) stated that moisture and nutrients have significant relationships with SMBC. The results showed that although SMBC under different tree species did not differ, soil microbial biomass are drived by different factors.

The $\mathrm{qCO}_{2}$ is a measure that varies according to the state of the microbial biomass, the availability of nutrients; and various abiotic factors (Wardle and Ghani, 1995; Gonçalves et al., 2009). qCO $\mathrm{C}_{2}$ showed a parallel change between plots and reached its highest level in September. This situation can be explained as in this period, with moisture and temperature changes (low moisture and high temperature) as it was stated by Yuan and Yue (2012). In addition, $\mathrm{SqCO}_{2}$ has generally changed at high levels in $\mathrm{A}_{\mathrm{n}}$ plot. Increase of $\mathrm{qCO}_{2}$ was interpreted as a microbial response to adverse environmental stresses that was observed when the soil conditions were unfavorable (Wardle and Ghani, 1995). $\mathrm{SqCO}_{2}$ had a positive relationship with $\mathrm{N}$ and $\mathrm{SM}$ in the $\mathrm{A}_{\mathrm{b}}$ plots, had a negative relationship with SMBC in the $\mathrm{A}_{c}$ plot and had a positive relationship with ST and EC, and had a negative relationship with SM in the $A_{n}$ plot. These results show that moisture is the limiting factor in $A_{n}$ plot and microbial biomass plays a role as the limiting factor in $\mathrm{A}_{\mathrm{c}}$ plots.

\section{Forest floor}

In this study, FFMR ranged from 0.41 to $9.24 \mu \mathrm{g} \mathrm{CO}_{2}-\mathrm{C} \mathrm{g}^{-} \mathrm{h}^{-}$. These values are lower than the values found for fir forests by Bolat et al. (2015). Although the a mean FFMR values did not differ significantly among the species, forest floor properties (FFC, FFCN, FF and K) differed among plots. FFMR in all 
plots was observed as it decreased in the autumn period and an increase afterwards. This can be explained by the change in the amounts of FMBC. In relation to this, when the relationships among the variables were evaluated, it showed a linear correlation between FFMR and FFMBC in all plots. In addition, FFMR had a positive correlation with $\mathrm{Ca}$ in the $\mathrm{A}_{\mathrm{b}}$ plot, a positive relationship with $\mathrm{FM}$ and a negative relationship with $\mathrm{N}, \mathrm{C}$ and $\mathrm{P}$ in the $\mathrm{A}_{\mathrm{n}}$ plot. Also, it has shown the only negative relationship with $\mathrm{P}$ in the $\mathrm{A}_{\mathrm{c}}$ plot. Wardle (1992) stated that microbial activity may also be limited by the availability of $\mathrm{N}$ or P. Contrary to the presented study results, Allen and Schlesinger (2004) found a linear correlation between FFMR and C, N, P. As a result, nutrient status may also affect the activity of the microflora.

Microbial biomass mediates the conversion of biogenic nutrients between inorganic and organic components (Wu et al., 2000). FFMR values were similar and there was no difference between the plots in terms of average FFMBC amounts. It had decreased from spring to autumn in terms of the temporal change. This variation can be caused by changes in temperature and humidity. Similar to the study results, Butenschoen et al. (2011) found that the litter microbial biomass decreases with increasing temperature. In addition, it is possible to say that the mobilization and immobilization status of nutrients can be temporally effective. As a matter of fact, when the relationships between FFMBC and variables are evaluated, there was no positive relationship in the $A_{b}$ and $A_{c}$ plot while there was a positive relationship with $\mathrm{K}$ in the $\mathrm{A}_{\mathrm{n}}$ plot. On the contrary, the results showed a negative relationship with $\mathrm{Na}$ in $\mathrm{A}_{\mathrm{b}}$ plot, with $\mathrm{P}$ in $\mathrm{A}_{\mathrm{c}}$ plot and with $\mathrm{Ca}$ in $\mathrm{A}_{\mathrm{n}}$ plot. This observation shows that different nutrients may have a limiting effect on a variation of forest floor microbial biomass among the plots.

The mean values of $\mathrm{FFqCO}_{2}$ were not showed a significant difference among the plots like the other microbial parameters and it reached the highest level in November. This can be explained by the mobilization process of nutrients. Because the $\mathrm{FFqCO}_{2}$ showed a negative relationship with $\mathrm{K}$ in the $\mathrm{A}_{\mathrm{b}}$ plot, with $\mathrm{P}$ in the $\mathrm{A}_{\mathrm{c}}$ plot, and with $\mathrm{Ca}$ in the $\mathrm{A}_{\mathrm{n}}$ plot. This result supports the view that the limiting factors mentioned above were caused by different nutrients.

\section{Conclusion}

In the presented study, $\mathrm{SMBC}, \mathrm{SqCO}_{2}$ values did not show a significant difference among the three different fir species, and SMR has the lowest value in Abies nordmanniana ssp. bornmuelleriana Mattf. plot. While SMR and SMBC were associated with moisture and temperature conditions in the Abies nordmanniana ssp nordmanniana Mattf plot, they had interactions with nutrients ( $\mathrm{N}, \mathrm{Na}, \mathrm{K}$ and $\mathrm{P}$ ) in other plots. Microbial parameters in forest floor did not differ between plots. Temporarily, a decrease in microbial parameters have been detected from spring to autumn in plots. Forest floor microbial parameters prominently changed under the influence of nutrients $(\mathrm{Ca}, \mathrm{N}, \mathrm{C}, \mathrm{P}$ and $\mathrm{K})$. As a result, in this short-term study, although there was no clear difference between the plots, the microbial processes in both soil and forest floor changed with the effect of different factors. These results will contribute to understanding the relationships between microbial processes and biochemical cycles in plantations. In future studies, considering the long-term measurements of these interrelations, including microbial community structure and enzyme activities, will also provide a clearer evaluation opportunity in plantations constituted different species.

\section{Acknowledgements}

This work was supported by the Scientific Research Projects Coordination Unit of Istanbul University-Cerrahpasa, Project number: BYP-2017-23685. It was presented as a oral presentation titled "Doğu Karadeniz ve Uludağ Göknarı Meşcereleri Altındaki Topraklarda Bazı Mikrobiyal Parametrelerin Mevsimsel Değişimi”, and its abstract (Turkish) was published in the abstracts book of the Congress "22. Ulusal Biyoloji Kongresi" in Eskisehir, Turkey, 23-27 June 2014. I thank to Res. Assis. Alper Gün ÖZTURNA for his support with English language editing. 


\section{References}

Agnelli, A., Ugolini, F., Corti, G., Pietramellara, G. (2001). Microbial biomass-C and basal respiration of fine earth and highly altered rock fragments of two forest soils. Soil Biology and Biochemistry 33, 613-620.

Akburak, S., Son, Y., Makineci, E., Çakir, M. (2018). Impacts of low-intensity prescribed fire on microbial and chemical soil properties in a Quercus frainetto forest. Journal of forestry research 29, 687-696.

Alef, K., Nannipieri, P., 1995. Methods in applied soil microbiology and biochemistry. Academic Press.

Allen, A., Schlesinger, W. (2004). Nutrient limitations to soil microbial biomass and activity in loblolly pine forests. Soil Biology and Biochemistry 36, 581-589.

Anderson, T.H., Domsch, K. (1986). Carbon assimilation and microbial activity in soil. Zeitschrift für Pflanzenernährung und Bodenkunde 149, 457-468.

Araujo, A., Silva, E., Nunes, L., Carneiro, R. (2010). The effect of converting tropical native savanna to Eucalyptus grandis forest on soil microbial biomass. Land degradation \& development 21, 540-545.

Baldrian, P. (2017). Microbial activity and the dynamics of ecosystem processes in forest soils. Current opinion in microbiology $37,128-134$.

Bolat, I. (2014). The effect of thinning on microbial biomass $\mathrm{C}, \mathrm{N}$ and basal respiration in black pine forest soils in Mudurnu, Turkey. European journal of forest research 133, 131-139.

Bolat, İ., Kara, Ö., Tunay, M. (2015). Effects of Seasonal Changes on Microbial Biomass and Respiration of Forest Floor and Topsoil under Bornmullerian Fir Stand. Eurasian Journal of Forest Science 3, 1-13.

Brookes, P., Cayuela, M.L., Contin, M., De Nobili, M., Kemmitt, S., Mondini, C. (2008). The mineralisation of fresh and humified soil organic matter by the soil microbial biomass. Waste Management 28, 716-722.

Butenschoen, O., Scheu, S., Eisenhauer, N. (2011). Interactive effects of warming, soil humidity and plant diversity on litter decomposition and microbial activity. Soil Biology and Biochemistry 43, 1902-1907.

Cheng, F., Peng, X., Zhao, P., Yuan, J., Zhong, C., Cheng, Y., Cui, C., Zhang, S. (2013). Soil microbial biomass, basal respiration and enzyme activity of main forest types in the Qinling Mountains. PLoS One 8.

Çakır, M. (2018). Richness and diversity of litter and soil fauna as affected by differences in three fir species. Bosque 39, 441-447.

Çakır, M., Akburak, S. (2017). Litterfall and nutrients return to soil in pure and mixed stands of oak and beech. İstanbul Üniversitesi Orman Fakültesi Dergisi 67, 185-200.

Diaz-Ravina, M., Acea, M., Carballas, T. (1993). Seasonal fluctuations in microbial populations and available nutrients in forest soils. Biology and Fertility of Soils 16, 205-210.

Docherty, K.M., Borton, H.M., Espinosa, N., Gebhardt, M., Gil-Loaiza, J., Gutknecht, J.L., Maes, P.W., Mott, B.M., Parnell, J.J., Purdy, G. (2015). Key edaphic properties largely explain temporal and geographic variation in soil microbial communities across four biomes. PLoS One 10. 
Glassman, S.I., Weihe, C., Li, J., Albright, M.B., Looby, C.I., Martiny, A.C., Treseder, K.K., Allison, S.D., Martiny, J.B. (2018). Decomposition responses to climate depend on microbial community composition. Proceedings of the National Academy of Sciences 115, 11994-11999.

Gonçalves, I., Araújo, A., Carvalho, E., Carneiro, R. (2009). Effect of paclobutrazol on microbial biomass, respiration and cellulose decomposition in soil. European journal of soil biology 45, 235-238.

Haripal, K., Sahoo, S. (2014). Microbial biomass Carbon, Nitrogen, and Phosphorus dynamics along a chronosequence of abandoned tropical agroecosystems. International Journal of Current Microbiology and Applied Sciences 3, 956-970.

Hofman, J., Dušek, L., Klánová, J., Bezchlebová, J., Holoubek, I. (2004). Monitoring microbial biomass and respiration in different soils from the Czech Republic - a summary of results. Environment International 30, 1930.

Jia, G.-m., Cao, J., Wang, C., Wang, G. (2005). Microbial biomass and nutrients in soil at the different stages of secondary forest succession in Ziwulin, northwest China. Forest Ecology and Management 217, 117-125.

Jiang, J.-P., Xiong, Y.-C., Jiang, H.-M., De-You, Y., Ya-Jie, S., Feng-Min, L. (2009). Soil microbial activity during secondary vegetation succession in semiarid abandoned lands of Loess Plateau. Pedosphere 19, 735-747.

Kara, Ö., Bolat, I. (2008). Soil microbial biomass C and $\mathrm{N}$ changes in relation to forest conversion in the Northwestern Turkey. Land Degradation \& Development 19, 421-428.

Li, Y., Liu, Y., Wu, S., Niu, L., Tian, Y. (2015). Microbial properties explain temporal variation in soil respiration in a grassland subjected to nitrogen addition. Scientific reports 5, 18496.

Mariani, L., Chang, S.X., Kabzems, R. (2006). Effects of tree harvesting, forest floor removal, and compaction on soil microbial biomass, microbial respiration, and $\mathrm{N}$ availability in a boreal aspen forest in British Columbia. Soil Biology and Biochemistry 38, 1734-1744.

Nsabimana, D., Haynes, R., Wallis, F. (2004). Size, activity and catabolic diversity of the soil microbial biomass as affected by land use. Applied Soil Ecology 26, 81-92.

OGM, 2013. Orman Atlası. Orman Genel Müdürlüğü, Ankara.

Oktay, S., Tecimen, B.H. (2016). Nitrogen Mineralization Experiment under Fir Stand with Different Measurement Methods Asian Journal of Agriculture and Food Sciences 4, 157-163.

Oyedele, A.O., Olayungbo, A.A., Denton, O.A., Ogunrewo, O.M., Momodu, F.O. (2015). Assessment of the microbial biomass carbon, nitrogen and phosphorus in relation to physico-chemical properties of Acric Luvisols in Ibadan South West, Nigeria. Journal of Agriculture and Environment for International Development (JAEID) 109, 179-187.

Park, J.-H., Kalbitz, K., Matzner, E. (2002). Resource control on the production of dissolved organic carbon and nitrogen in a deciduous forest floor. Soil Biology and Biochemistry 34, 813-822.

Pei, Z., Eichenberg, D., Bruelheide, H., Kröber, W., Kühn, P., Li, Y., von Oheimb, G., Purschke, O., Scholten, T., Buscot, F. (2016). Soil and tree species traits both shape soil microbial communities during early growth of Chinese subtropical forests. Soil Biology and Biochemistry 96, 180-190. 
Pietri, J.A., Brookes, P. (2008). Relationships between soil pH and microbial properties in a UK arable soil. Soil Biology and Biochemistry 40, 1856-1861.

Prescott, C.E., Grayston, S.J. (2013). Tree species influence on microbial communities in litter and soil: current knowledge and research needs. Forest Ecology and Management 309, 19-27.

Priess, J., Fölster, H. (2001). Microbial properties and soil respiration in submontane forests of Venezuelian Guyana: characteristics and response to fertilizer treatments. Soil Biology and Biochemistry 33, 503-509.

Qu, L., Kitaoka, S., Koike, T. (2018). Factors controlling soil microbial respiration during the growing season in a mature larch plantation in Northern Japan. Journal of soils and sediments 18, 661-668.

Schoenholtz, S.H., Van Miegroet, H., Burger, J. (2000). A review of chemical and physical properties as indicators of forest soil quality: challenges and opportunities. Forest ecology and management 138, 335-356.

Singh, J. (2018). Microbes: Key Ecological Drivers in Controlling the Issues Related to Environmental Changes. Annals of Microbiology and Immunology 1, 1009.

Soong, J.L., Marañon-Jimenez, S., Cotrufo, M.F., Boeckx, P., Bodé, S., Guenet, B., Peñuelas, J., Richter, A., Stahl, C., Verbruggen, E. (2018). Soil microbial CNP and respiration responses to organic matter and nutrient additions: Evidence from a tropical soil incubation. Soil Biology and Biochemistry 122, 141-149.

Spohn, M. (2015). Microbial respiration per unit microbial biomass depends on litter layer carbon-to-nitrogen ratio. Biogeosciences 12, 817-823.

Tan, X., Chang, S.X., Kabzems, R. (2008). Soil compaction and forest floor removal reduced microbial biomass and enzyme activities in a boreal aspen forest soil. Biology and Fertility of Soils 44, 471-479.

Wang, Q., Kwak, J.-H., Choi, W.-J., Chang, S.X. (2018). Decomposition of trembling aspen leaf litter under longterm nitrogen and sulfur deposition: effects of litter chemistry and forest floor microbial properties. Forest Ecology and Management 412, 53-61.

Wang, W., Dalal, R., Moody, P., Smith, C. (2003). Relationships of soil respiration to microbial biomass, substrate availability and clay content. Soil Biology and Biochemistry 35, 273-284.

Wardle, D. (1992). A comparative assessment of factors which influence microbial biomass carbon and nitrogen levels in soil. Biological reviews 67, 321-358.

Wardle, D., Ghani. (1995). A critique of the microbial metabolic quotient (qCO2) as a bioindicator of disturbance and ecosystem development. Soil Biology and Biochemistry 27, 1601-1610.

Wolters, V., Joergensen, R. (1991). Microbial carbon turnover in beech forest soils at different stages of acidification. Soil Biology and Biochemistry 23, 897-902.

Wu, J., He, Z.-L., Wei, W.-X., O'donnell, A., Syers, J. (2000). Quantifying microbial biomass phosphorus in acid soils. Biology and Fertility of Soils 32, 500-507. 
Yang, K., Zhu, J., Zhang, M., Yan, Q., Sun, O.J. (2010). Soil microbial biomass carbon and nitrogen in forest ecosystems of Northeast China: a comparison between natural secondary forest and larch plantation. Journal of Plant Ecology 3, 175-182.

Yuan, B.-C., Yue, D.-X. (2012). Soil microbial and enzymatic activities across a chronosequence of Chinese pine plantation development on the loess plateau of China. Pedosphere 22, 1-12.

Submitted: 02.03.2020 Accepted: 14.04.2020 